\title{
CTR MEETS REALITY
}

\author{
$M$ Ibrahim Khan ${ }^{*}$
}

'Orthopedic \& Hand surgery, Beverly Hills, CA, USA

\section{Article Info}

\section{Article Notes}

Received: September 27, 2017

Accepted: January 10, 2018

\section{${ }^{*}$ Correspondence:}

Dr. M Ibrahim Khan

Orthopedic \& Hand surgery, Beverly Hills, CA, USA, Email: mibrahimkhanmd7@aol.com

(c) $2018 \mathrm{Khan} \mathrm{Ml}$. This article is distributed under the terms of the Creative Commons Attribution 4.0 International License
I applaud Logan McCool et al for "A review of advances in carpal tunnel release" ${ }^{1}$ but I must point out that these innovations have not made an iota of difference in the outcome of carpal tunnel release (CTR) which remains suboptimal. The cascading effects of this is on display in the study by Menendez et $\mathrm{al}^{2}$ which has prompted a mad scramble for the solution that was quite palpable at the annual meeting of American Society for Surgery of hand (ASSH) in Sept; 2017 in San Francisco where an instructional coarse (IC 46) on: Challenges in the treatment of carpal tunnel syndrome, there were 4 presentations on: The unhappy patient after CTR. But what was presented was same ole, same ole.

To solve this problem we need 'new knowledge' and the clue to this 'new knowledge' can be found in this study from Mayo clinic: Pre- \& postoperative dynamic ultrasound assessment of the median nerve in patients undergoing CTR; which was read at the 2017 EUROHAND in Budapest by Evers et al ${ }^{3}$ " Although CTR is effective in reducing pressure, $70-90 \%$ of the patients have residual symptoms. Previous studies have shown that the shape and motion patterns of the median nerve are altered in patients with CTS compared to controls. These parameters might also indicate whether CTR is effective. The aim of this study was to sonographically assess the effectiveness of CTR on median nerve deformation and excursion." What they found that there was no change in the dynamic measurement of the transverse sliding of median nerve, in other words it remained restricted as it was before. Although the authors were blindsided by this finding, I'm not. More on this later.

As physicians we should be mindful that in science, more often than not, theories are not rejected because they were tested and proved wrong in the lab, they are rejected because of bad explanations. After all, scientific explanations are about reality. CTS as a compressive neuropathy is the poster child for bad explanations. Case in point. The fact that compression theory cannot square the natural history circle of CTS has unleashed a series of negative consequences like the illusion of 'Hot CTS ' and 'Cold CTS' and the illusion that carpal tunnel release (CTR) works best in 'Hot CTS'4 not to mention the electro diagnostic (EDX) riddle of $\mathrm{CTS}^{5}$ which is the result of the lack of concordance between patient symptoms and the EDx findings and the assumption of an irremediable binary choice between the symptoms and the EDx findings as rationalization of sub optimal outcome of $\mathrm{CTR}^{6}$.

It's not that the natural history of CTS is unknowable as the editorin-chief of the journal of hand surgery (American edition) would have us believe ${ }^{7}$ it is that we are looking at CTS through a distorted lens of compression.

Coming back to the restricted transverse sliding of median nerve 

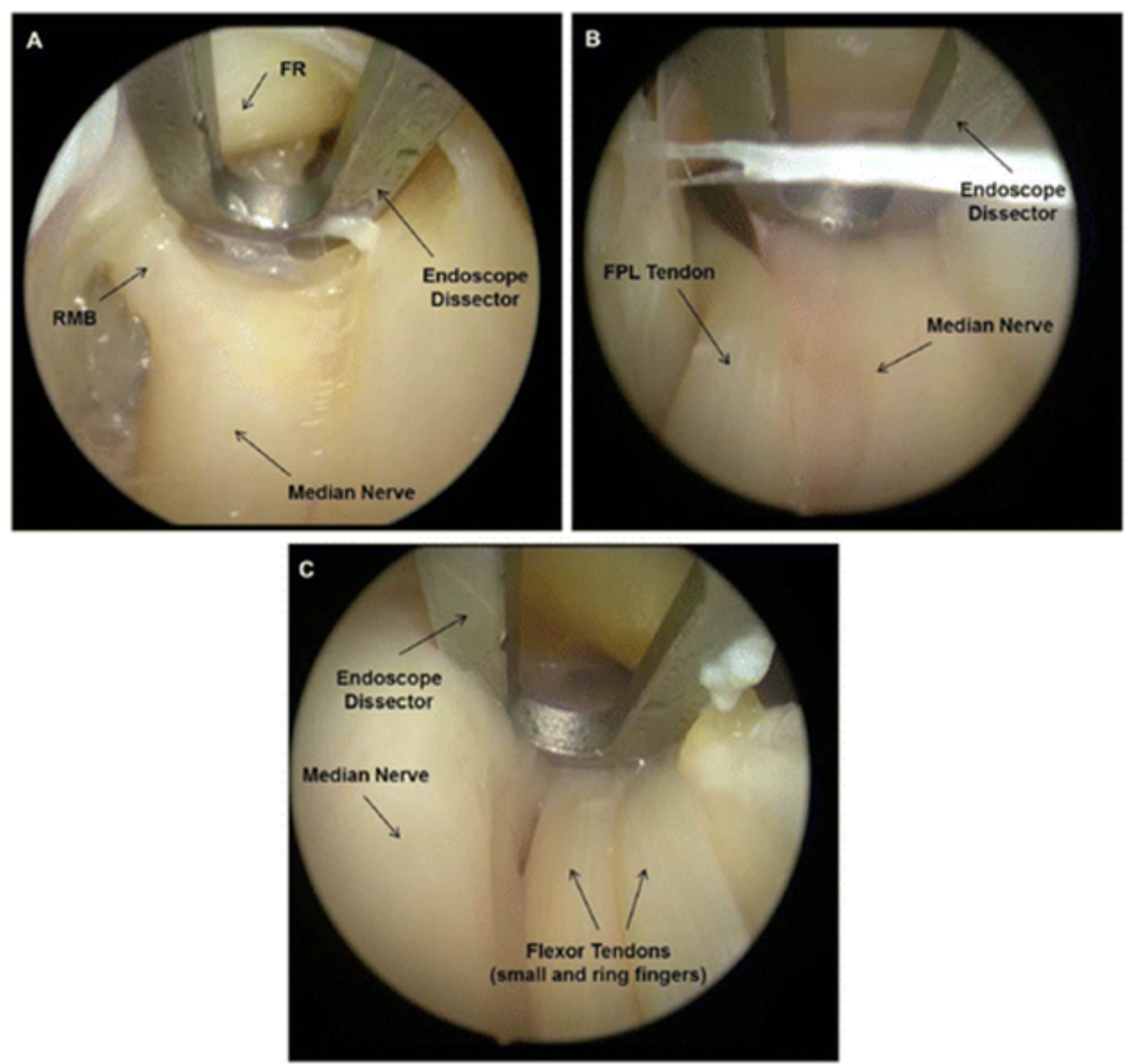

Figure1. (A) View of the median nerve, flexor retinaculum (FR), recurrent motor branch (RMB), \& endoscope dissector. (B, C) flexor pollicis longus (FPL) tendon \& flexor tendons to the small \& ring fingers. Note: The loss of normal physiological space between the median nerve \& FPL in figure 1B in stark contrast to figure 1C where there's no loss of this space between the median nerve \& the ulnar synovial bursa with enclosed flexor tendons.(Figure1 in my letter to the editor, permission to republish obtained).

in carpal tunnel which remained unchanged after CTR in the aforementioned study from Mayo clinic. This finding in idiopathic CTS was first reported by Nakamichi \& Tashibana in $1995^{\circ}$ but it gained no traction because it was not explainable on the basis of compression theory for CTS. But now it is fully explainable on the basis of subtle, but crucial, dynamic median neurodesis to flexor pollicis longus (FPL) in carpal tunnel which I discovered serendipitously during open carpal tunnel surgery in 1997 and endoscopic proof of that is in these jaw-dropping images of Idiopathic CTS (Figure 1) ${ }^{9}$. This new pathophysiology for idiopathic CTS helps us see the big picture by putting everyone on the same page thus linking the aesthetic truth of what the patient is experiencing (symptoms) to the factual ones in the sonographic \& EDx findings thus restoring full concordance between the symptoms of the patient and the sonographic \& EDx findings thereby nixing the EDx riddle of CTS while simultaneously cancelling the so called irremediable binary choice between the symptoms and the EDx findings and hence bringing an end to all excuses for a sub optimal outcome of carpal tunnel surgery. This neurodesis culminates in CTS on the basis of 'traction neuropathy' and not 'compression neuropathy'. The dynamics of this neurodesis come into play during gripping because of reciprocal; divergent; translational; relationship (RDTR) between FPL \& median nerve. We can understand that during gripping, the FPL has to move radially to get in line with the thumb and dorsally to get closer to the bone $\&$ joint to become an efficient \& effective thumb flexor. The median nerve moves in the opposite direction i.e. ulnarly $\&$ volarly. But in the presence of this neurodesis, the nerve can't move. So, this restricted transverse sliding of the median nerve in carpal tunnel sets in motion a cascade of inevitable morphological \& physiological changes in the median nerve which on one side determine the symptoms of the patient and on the other side determine the sonographic findings such as enlarged cross sectional area (CSA) of median nerve, decreased echogenicity of median nerve as well as findings of the electro diagnostic examination (EDx).

This is how the CSA gets enlarged. As the nerve is unable to slide transversely, this puts the nerve on a stretch \& increased tension. And this stretching of the nerve is exactly what results in enlarged CSA as Nakamichi \& Tachibana had expected in the prescient conclusion of 
their 2000 seminal paper on enlarged CSA: "We conclude that idiopathic CTS is characterized by severity-correlated intracarpal enlargement of the median nerve, not by compressive deformation, such as a reduction in the caliber of the nerve. This enlargement may have a role in disease pathophysiology. ${ }^{10}$.

By the same token, there will be decreased echogenicity of the median nerve because of thinning resulting from stretching (imagine a piece of cloth being stretched) and consequently fewer nerve fibers per unit area. This decreased echogenicity like enlarged CSA refutes CTS as a compressive neuropathy because in compression echogenicity of the nerve will be increased like the picture that we see in neurofibroma and not decreased. Furthermore as this stretching remains uncorrected over prolonged period of time, the attenuated median nerve becomes the center of the hour glass appearance that we have become accustomed to seeing in advanced cases of Idiopathic CTS. Finally, what about the claim that CTR works best in " Hot CTS"? ${ }^{4}$ I have analyzed this claim in detail else where ${ }^{9}$ suffice here to state that this claim is nothing more than the proverbial rooster taking credit for the sun rise.

\section{References}

1. McCool L, Kliot M, Guo D. A Review of Advances in Carpal Tunnel Release. J Neurol Neuromed 2017; 2(10): 6-8

2. Menendez ME, Chen NC, Chaitranya SM, et al. Physician empathy as a driver of hand surgery patient satisfaction. J Hand Surg Am. 2015; 40 (9): 1860-1865.

3. Evers S. et al Pre- \& postoperative dynamic ultrasound assessment of the median nerve in patients undergoing CTR. J Hand surg Eur. June 2017; issue1_suppl: FESSH abstracts.

4. Gelberman RH. The numb hand: how I approach carpal tunnel syndrome. AAOS $72^{\text {nd }}$ annual meeting Feb 2005; 23-27. WashingtonDC

5. Padua L, Padua R, Lo Monaco M, et al. Natural history of carpal tunnel syndrome according to the neurophysiological classification. Italian journal of Neural sciences. 1998; (6): 357-61.

6. Yang CQY, Lai SWH, Tay SC. Long-term outcome of carpal tunnel release surgery in patients with severe carpal tunnel syndrome. BJJ 2017; 99-B (10): 1348-1353.

7. Graham B. Evidence-Based medicine: Non-surgical treatment of carpal tunnel syndrome. J Hand Surg. 2009; 34 A: 531-534.

8. Nakamichi K, Tachibana S. Restricted motion of the median nerve in carpal tunnel syndrome. J Hand Surg (Br). August 1995; 20: 460-464.

9. Khan MI. CTS: Reality Check. J Trauma care. 2017; 3(3): 1025.

10. Nakamichi K, Tachibana S. Enlarged median nerve in idiopathic carpal tunnel syndrome. Muscle \& Nerve. 2000; 23: 1713-1718. 